\title{
Schreiben, wie Godard Filme macht
}

\author{
PRÄLIMINARIEN EINES INTERMEDIALEN EDITIONSPROJEKTS
}

Wolfgang Straub

Robert-Musil-Institut für Literaturforschung / Kärntner Literaturarchiv
wolfgang.straub@aau.at

Keywords: Intermediality, Werner Kofler, Hybrid Edition, Edition Philology, Austrian

Literature, Digital Edition

\begin{abstract}
Based on the oeuvre of the Austrian writer Werner Kofler (1947-2011) the article focusses on the question how intermediality can be situated in the field of Digital Humanities.
\end{abstract}

Als Roman Jakobson 1959 seinen berühmt gewordenen Vortrag über die Zusammenhänge von Linguistik und Poetik hielt, war für inn und sein Publikum der Gedanke an die "Ilias" oder "Odyssee" als Comic ein lächerlicher. ${ }^{1}$ Heute ist uns dieser Gedanke ein vertrauter, mehr oder weniger die halbe kanonisierte Weltliteratur liegt als Comic oder Graphic Novel vor. Die Ausführungen des Semiotikers und Linguisten Jakobson zeugen davon, wie spätestens um die Mitte des 20. Jahrhunderts das LaokoonParadigma - die in Lessings Schrift geforderte Trennung von Text und Bild - auch in der Literaturwissenschaft seine Gültigkeit einzubüßen beginnt, nachdem zuvor den Avantgarden zu Beginn des 20. Jahrhunderts an medialen Grenzüberschreitungen zu tun gewesen war.

Jakobson spricht von einer „Vergleichbarkeit der verschiedenen Künste“. ${ }^{2}$ Es wird aber bis ins letzte Jahrzehnt des 20. Jahrhunderts dauern, bis die Intermedialität in den Mittelpunkt literaturwissenschaftlichen - und zu Beginn speziell des komparatistischen - Forschungsinteresses rückt; im Rückblick machte man gar einen „intermedial turn“ in der Mitte der 1990er Jahre fest. ${ }^{3}$ Vor dem Hintergrund einer auch im deutschsprachigen Raum ungebrochen umtriebigen Intermedialitätsforschung ${ }^{4}$ will sich mein Beitrag anhand eines anlaufenden literaturwissenschaftlichen Editionsprojekts mit der Frage beschäftigen, ob und wie sich Intermedialität als Forschungsgegenstand im Rahmen der digitalen Geisteswissenschaften positionieren (und fruchtbar machen) lässt.

1 Roman Jakobson, Linguistik und Poetik, in: Roman Jakobsen, Poesie der Grammatik und Grammatik der Poesie. Sämtliche Gedichtanalysen. Hrsg. von Hendrik Birus / Sebastian Donat, Bd. 1: Poetologische Schriften und Analysen zur Lyrik vom Mittelalter bis zur Aufklärung, Berlin / New York 2007, 155-216, hier 157.

2 Ebd.

3 Wolf gibt eine Konferenz im schwedischen Lund 1995, veranstaltet von der Komparatistin Ulla-Britta Lagerroth, als wichtigen Impulsgeber für die internationale Intermedialitätsforschung an. Vgl. Werner WoLF, Towards a Functional Analysis of Intermediality. The Case of Twentieth-Century Musicalized Fiction, in: Erik Hedling / Ulla-Britta Lagerroth, Hg., Cultural Functions of Intermedial Exploration, Amsterdam, New York 2002, 15-34, hier 15. Allg. zum „intermedial turn“ vgl. Christine SCHWANECKE, Filmic Modes in Literatur, in: Gabriele Rippl, Hg., Handbook Intermediality. Literatur - Image - Sound Music, Berlin / Boston 2015, 268-286, hier 272-279.

4 Vgl. u.a.: Uta DegneR / Norbert Christian Wolf, Hg., Der neue Wettstreit der Künste. Legitimation und Dominanz im Zeichen der Intermedialität, Bielefeld 2010; Evi ZEMANEK, Intermedialität - Interart Studies, in: Evi Zemanek / Alexander Nebrig, Hg., Komparatistik, Berlin 2012, 159-174; Dunja BRÖTz / Beate EDER-JoRDAN / Martin FRITZ, Hg., Intermedialität in der Komparatistik. Eine Bestandsaufnahme, Innsbruck 2013; Steven Tötösy de Zepetnek, Hg., Digital Humanities and the Study of Intermediality in Comparative Cultural Studies, Lavayette 2013; Stefan KEPPLER-TASAKI / Wolf Gerhard SCHMIDT, Hg., Zwischen Gattungsdisziplin und Gesamtkunstwerk. Literarische Intermedialität 1815-1848, Berlin 2015. 


\section{Was ist Intermedialität?}

Eine exakte Definition von Intermedialität ist naturgemäß nicht möglich. Das liegt bereits in der Unmöglichkeit begründet, einen eindeutigen Medienbegriff zu postulieren. ,Medium“ kann man, dem konventionellen Verständnis nach und Werner Wolf folgend, als distinkt wahrgenommenes Kommunikationsdispositiv verstehen. ${ }^{5}$ Jörg Robert umreißt, dabei eine erste Definition mitliefernd, den Schritt von der Medien-Definition zur Intermedialität als Bewegung ins Uferlose: „Die Schwierigkeiten mit dem Medienbegriff potenzieren sich im Hinblick auf die Intermedialität. Dies gilt ganz buchstäblich. Intermedialität ist die Bezeichnung für eine Relation zwischen zwei Medien. Beide müssen sich in einer gemeinsamen ,dritten' Ebene - einer als Universalie angenommenen ,Medialität" - begegnen." 6 Ein Begriff sowohl von Medien als auch von Intermedialität lässt sich nur diskursiv herleiten und ist daher "ebenso vielfältig wie die Diskurse, in denen er verwendet wird."

Trotz dieser unumgänglichen begrifflichen Vagheit haben sich nicht wenige Forscher*innen an einer Typologie der Intermedialität versucht. ${ }^{8}$ Die pragmatischste und klarste Darstellung stammt wohl von Irina Rajewsky, die sich dabei mit drei Phänomenen begnügt: Intermedialität im engeren Sinne umfasse einen Medienwechsel (z.B. Literaturverfilmungen); daneben gebe es die Medienkombination, wie sie etwa auf der Opernbühne, am Theater oder in illuminierten Handschriften auftrete; und schließlich seien in der Literatur vor allem die intermedialen Bezüge relevant, etwa bei der klassischen Ekphrasis oder wenn ein Text auf Film Bezug nehme oder wenn man von einer "Musikalisierung der Literatur" spreche. ${ }^{9}$

\section{Was bedeutet Intermedialität im digitalen Zeitalter?}

Für die Frage, wie Intermedialität sich in und mit den „Neuen Medien“ geriere, haben Bolter und Grusin im Jahre 2000 einen in der Folge vieldiskutierten Begriff geprägt: remediation. ${ }^{10}$ Von dieser Begriffsprägung ausgehend verweist der Medienwissenschaftler Jürgen E. Müller darauf, dass man es im Digitalen mit einer Fortsetzung intermedialer Prozesse auf „einem Niveau [zu tun hat], in dem Vorstellungen von Gattungen, Formaten, Funktionen in re-medialisierter oder recycelter Form mit dem Sinnbildungsvermögen des ,Users‘ spielen.“"11

Diese Remedialisierung spielt im derzeit nach wie vor breitesten Feld der digitalen Literaturwissenschaft eine große Rolle: Die digitalen Editionen bilden Bücher ab oder simulieren Bücher, und sie tragen das Literaturarchiv, verschiedenartig aufbereitet und kommentiert, ins Netz. Dabei spielt das WWW die Rolle der von Jörg Robert angesprochenen „dritten Ebene“. Anders verhält es sich, wenn

5 Vgl. Werner Wolf, Intermedialität: Ein weites Feld und eine Herausforderung für die Literaturwissenschaft, in: Herbert Foltinek / Christoph Leitgeb, Hg., Literaturwissenschaft intermedial - interdisziplinär, Wien 2002, 165.

6 Jörg ROBERT, Einführung in die Intermedialität, Darmstadt 2014, 22.

7 Irina O. RAJEWSKY, Intermedialität, light'? Intermediale Bezüge und die ,bloße Thematisierung des Altermedialen, in: Roger Lüdeke / Erika Greber, Hg., Intermedium Literatur. Beiträge zu einer Medientheorie der Literaturwissenschaft, Göttingen 2004, 27-76, hier 29.

8 Vgl. Jens SCHRÖTER, Discourses and Models of Intermediality, in: Tötösy de Zepetnek, Digital Humanities, 32-42; Werner Wolf, (Inter)mediality and the Study of Literature, in: Ebd., 19-31; Uwe WIRTH, Intermedialität, in: Alexander Roesler / Bernd Stiegler, Hg., Grundbegriffe der Medientheorie, München 2005, 114-121.

9 RAJEWSKY, Intermedialität, light'?, 37f.

10 Vgl. David Jay Bolter / Richard Grusin, Remediation. Understanding New Media, Cambridge/Mass. 2000; zur Kritik an Bolter / Grusin vgl. u.a. Thomas BECKER, Einleitung, in: Ders., Hg., Ästhetische Erfahrung in der Intermedialität, Bielefeld 2011, 7-32, hier 20f.

11 Jürgen E. MülLeR, Intermedialität digital. Konzepte, Konfigurationen, Konflikte, in: Andy Bläter u. a., Hg., Intermediale Inszenierungen im Zeitalter der Digitalisierung. Medientheoretische Analysen und ästhetische Konzepte, Bielefeld 2010, 17-39, hier 23. 
das Netz, etwa in der Medienkunst, Teil der intermedialen Interaktion selbst ist. Um beim Beispiel Literatur zu bleiben: Literatur im Netz bringt seit den 1990er Jahren eine eigenständige künstlerische Dimension ein („Netzliteratur“, „multimediale Literatur“), sie dynamisiert und visualisiert Ansätze, die man aus der analogen Literatur kennt, etwa Lautpoesie, konkrete oder visuelle Poesie. Sie macht aus dem Schriftsteller einen Programmierer - oder, im (plakativen) Sinne Kenneth Goldsmiths, einen Textprozessor, der das vorhandene Textmaterial (im Web) nur mehr zu einem weiteren Text organisiert. ${ }^{12}$ Und längst gibt es einen weiteren (Rück-)Schritt der Remedialisierung: In gedruckten Büchern werden digitale Kommunikationsformen abgebildet bzw. simuliert (E-Mail-, Twitter-, Facebook-Romane, gedruckte Blogs, etc.).

\section{3. „Kofler intermedial“}

Das Werk des österreichischen Schriftstellers Werner Kofler (1947-2011) ist prädestiniert wie wenige für eine Analyse intermedialer Phänomene. Kofler ist bekannt für seine satirische Prosa, er war aber auch einer der eifrigsten Hörspielautoren des Landes, er verfasste zwei Theaterstücke und setzte einen Kurzfilm als Regisseur um. Seine Literatur wird als „polyphon“ beschrieben, ihr wird zugesprochen, die „Medialisierung einer selbst schon durch und durch medialisierten Welt“13 zu leisten. Kofler selbst war über den Texttransfer („Mehrfachnutzung“) zwischen Prosa und Radio/Theater/Film hinaus dem Intermedialen zugetan, inm war Musik und Musikalität in seinen Texten von großer Bedeutung. Zudem schweben inm Filme als Vorbilder vor: In einer Vorbemerkung schreibt er von einem „Hörspiel zwischen Godards ,Weekend" und Fellinis ,Orchesterprobe “"'14, in seinen Notizen findet sich der Satz: „Schreiben, wie Godard Filme macht [/] (Es geht.)“15

Nachdem in einem FWF-Forschungsprojekt 2015-2018 die Prosa Koflers in einer kommentierten Hybridedition zugänglich gemacht wurde, ${ }^{16}$ geht nun das Projekt „Kofler intermedial“ mit dem Projektpartner ACDH Graz daran, eine digitale Grundlage zu schaffen für die Analyse von Intermedialität (wobei hier der Text das Ausgangsmedium bleibt). Dabei machen wir uns den Umstand zunutze, dass grundlegende Fragen digitaler Editorik vom jeweiligen Medium unabhängig sind - Roland S. Kamzelak meint etwa, dass „die editorische Arbeit unabhängig von der Quelle gleich“ sei, „sie ist potentiell intermedial." ${ }^{17}$

Wir werden eine Forschungsplattform schaffen, auf der möglichst viele der Radio- und Filmarbeiten sowie Videoaufzeichnungen von Theateraufführungen zur Verfügung stehen. Eine besondere Herausforderung ist dabei der Umstand, dass die Veröffentlichung der Texte Koflers dem Urheberrecht unterliegen. Die Radioarbeiten werden zum Anhören auf der Plattform veröffentlicht, die (aus dem Nachlass generierten) Texte nur in einer analogen Buchversion zugänglich sein. Unsere „Mediensynopse sub auspiciis juris auctoris“ wird also viel mit dem wissenschaftlichen Zitat arbeiten müssen.

12 Vgl. Kenneth GoldSMITH, Uncreative Writing. Sprachmanagement im digitalen Zeitalter, Berlin 2017.

13 Bernhard FETZ, Stimmen hören. Zu Werner Koflers Triptychon Am Schreibtisch, Hotel Mordschein, Der Hirt auf dem Felsen, in: Herbert J. Wimmer, Hg., Strukturen erzählen. Die Moderne der Texte, Wien 1996, 133-151, hier 141.

14 Werner KofLer, Die vier Jahrezeiten. Hörspiel. Typoskript, Nachlass Kofler, Robert-Musil-Institut für Literaturforschung / Kärntner Literaturarchiv (Klagenfurt), Signatur 11, Mappe W27.

15 Werner Kofler, Notizblock, in: Klaus Amann, Hg., Werner Kofler. Texte und Materialien, Wien 2000, 222-228, hier 224.

16 Vgl. www.wernerkofler.at (15.04.2019).

17 Roland S. KAMZELAK, Mind Mapping (Musik)edition. Versuch einer Zusammenschau, in: Peter Stadler / Joachim Veit, Hg., Digitale Edition zwischen Experiment und Standardisierung. Musik - Text - Codierung, Tübingen 2009 (Beihefte zu editio), 233-236, hier 233. 
Ein Vorteil der Limitation durch das Urheberrecht ist die leichtere Einhaltung der Grenze zwischen den beiden Wissensformationen Archiv und Edition, die Rüdiger Nutt-Kofoth zu Recht eingefordert hat. ${ }^{18}$ Das Archiv (der Nachlass) ist für uns Wissensspeicher für die Erstellung des - im TEI-P5-Standard annotierten und mit Normdaten angereicherten - Stellenkommentars sowie die editorische Entscheidung für die jeweils autorisierte Textversion. Textgenetik ist kein primäres Forschungsinteresse. Bei Kofler sind intramediale Phänomene wie Systemerwähnung (z.B. Ekphrasis, Werberhetorik) oder Systemkontamination („musikalisches Schreiben“), ${ }^{19}$ die am Text untersucht werden können, von großer Bedeutung. Die Innovation von „Kofler intermedial“ wird die Herstellung einer Mediensynopse sein, die es ermöglicht, die Transposition von Textelementen über Mediengrenzen hinweg nachzuvollziehen und damit Formen (und idealerweise auch Funktionen) der Intermedialität zu erschließen. Damit entsteht - mittels ELAN-Annotation und IIIF-Transformation - eine Grundlage für eine intermediale Analyse der Text-Bild- und Text-Akustik-Zusammenhänge.

Digital Humanities sind per se interdisziplinär und intermedial, was aber nicht automatisch bedeutet, dass die Intermedialitätsforschung in den $\mathrm{DH}$ besonders stark vertreten wäre. Sie kann sich zwar „das Internet“ als „Universalmedium“ zunutze machen - ein Medium, das „alle bisherigen analogen Medien auf einer gemeinsamen technischen Basis und aus einem endlichen Vorrat diskreter Zeichen mit Algorithmen simulieren und somit beliebig austauschbar und verknüpfbar machen" ${ }^{20}$ kann. Aber diese „Austauschbarkeit“ wird selten thematisiert; die Verknüpfungen werden allerdings seit dem Aufkommen von Hyperlinks und „Hypermedia“ intensiv zur Vermittlung verwendet, wie sich etwa an dem am MIT entwickelten interaktiven Film „Berliner sehen“ (1995) zeigt. ${ }^{21}$ Kurt Fendt, leitender Entwickler dieses Spracherwerbstools, sieht zwanzig Jahre später in der Online-Intermedialität noch großes Potential. ${ }^{22}$ Wir hoffen, mit unseren literaturwissenschaftlichen Fragestellungen sowohl epistemologisch als auch in der Vermittlung beitragen zu können, die Intermedialitätsforschung in den DH zu stärken. Vielleicht nähern wir uns auf dem Feld der Intermedialität als Literaturwissenschaftler*innen jenem Bild an, das Heimito von Doderer 1957 vom Romancier zeichnete: „Dem Romancier stehen alle Fachwissenschaften zu Gebote, aber er muss möglichst lang Fachwissenschaftler bleiben." ${ }^{23}$

\section{Literaturverzeichnis}

Thomas BeCKER, Einleitung, in: Ders., Hg., Ästhetische Erfahrung in der Intermedialität, Bielefeld 2011, 7-32.

David Jay Bolter / Richard Grusin, Remediation. Understanding New Media, Cambridge/Mass. 2000.

Dunja Brötz / Beate EdER-JORDAN / Martin FRITZ, Hg., Intermedialität in der Komparatistik. Eine Bestandsaufnahme, Innsbruck 2013.

Uta DegneR / Norbert Christian Wolf, Hg., Der neue Wettstreit der Künste. Legitimation und Dominanz im Zeichen der Intermedialität, Bielefeld 2010.

18 Rüdiger NutT-Kоғотн, Edition als Archiv? in: Klaus Kastberger / Stefan Maurer / Christian Neuhuber, Hg., Schauplatz Archiv. Objekt - Narrativ - Performanz, Berlin 2019 (Literatur und Archiv 3), 107-124, hier $117 f$.

19 Vgl. Irina RAJEWSKY, Intermedialität, Tübingen / Basel 2002, 83-149.

20 Jörg SCHÄFER, Sprachzeichenprozesse. Überlegungen zur Codierung von Literatur in „alten“ und „neuen“ Medien, in: Jens Schröter / Alexander Böhnke, Hg., Analog/Digital - Opposition oder Kontinuum? Zur Theorie und Geschichte einer Unterscheidung, Bielefeld 2004, 143-168, hier 147.

21 Vgl. http://berlinersehen.mit.edu (15.04.2019).

22 Fendt sieht besonders jenen „Multimedia-Annotierunsgstools“ viel Potential, „die alle über gemeinsame Schnittstellen miteinander kommunizieren [...] und so Archive und Bibliotheken zu so genannten ,layered archives““ werden lassen können. Markus NeuschäFER, Perspektiven der Digital Humanities. Ein Interview mit Kurt Fendt, in: DHd-Blog, 12.10.2015, online unter: https://dhd-blog.org/?p=5735 (15.04.2019).

23 Heimito von Doderer im Gespräch mit Heinz Fischer Karwin [1957]. Nachzuhören auf: Heimito von Doderer $1896-1966$. Das Original. 3 CDs. Radio Österreich 1 „Edition Radio Literatur“, 1996, CD 1, Track 1. 
Heimito von Doderer im Gespräch mit Heinz Fischer Karwin [1957]. Nachzuhören auf: Heimito von Doderer 1896-1966. Das Original. 3 CDs. Radio Österreich 1 „Edition Radio Literatur“, 1996, CD 1, Track 1.

Bernhard FETZ, Stimmen hören. Zu Werner Koflers Triptychon Am Schreibtisch, Hotel Mordschein, Der Hirt auf dem Felsen, in: Herbert J. Wimmer, Hg., Strukturen erzählen. Die Moderne der Texte, Wien 1996, 133-151.

Kenneth GoldSMITH, Uncreative Writing. Sprachmanagement im digitalen Zeitalter, Berlin 2017.

Roman JakoBson, Linguistik und Poetik, in: Roman Jakobsen, Poesie der Grammatik und Grammatik der Poesie. Sämtliche Gedichtanalysen. Hrsg. von Hendrik Birus / Sebastian Donat, Bd. 1: Poetologische Schriften und Analysen zur Lyrik vom Mittelalter bis zur Aufklärung, Berlin / New York 2007, 155-216.

Roland S. KamzelaK, Mind Mapping (Musik)edition. Versuch einer Zusammenschau, in: Peter Stadler / Joachim Veit, Hg., Digitale Edition zwischen Experiment und Standardisierung. Musik - Text - Codierung, Tübingen 2009 (Beihefte zu editio), 233-236.

Stefan KePPLER-TASAKI / Wolf Gerhard SCHMIDT, Hg., Zwischen Gattungsdisziplin und Gesamtkunstwerk. Literarische Intermedialität 1815-1848, Berlin 2015.

Werner KofLER, Die vier Jahrezeiten. Hörspiel. Typoskript, Nachlass Kofler, Robert-Musil-Institut für Literaturforschung / Kärntner Literaturarchiv (Klagenfurt), Signatur 11, Mappe W27.

Werner Kofler, Notizblock, in: Klaus Amann, Hg., Werner Kofler. Texte und Materialien, Wien 2000, 222-228.

Jürgen E. MülleR, Intermedialität digital. Konzepte, Konfigurationen, Konflikte, in: Andy Bläter u. a., Hg., Intermediale Inszenierungen im Zeitalter der Digitalisierung. Medientheoretische Analysen und ästhetische Konzepte, Bielefeld 2010, 17-39.

Markus NeuschäFeR, Perspektiven der Digital Humanities. Ein Interview mit Kurt Fendt, in: DHd-Blog, 12.10.2015, online unter: https://dhd-blog.org/?p=5735 (15.04.2019).

Rüdiger Nutт-Kоғотн, Edition als Archiv? in: Klaus Kastberger / Stefan Maurer / Christian Neuhuber, Hg., Schauplatz Archiv. Objekt - Narrativ - Performanz, Berlin 2019 (Literatur und Archiv 3), 107-124.

Irina O. RAJEWSKY, Intermedialität, light'? Intermediale Bezüge und die ,bloße Thematisierung' des Altermedialen, in: Roger Lüdeke / Erika Greber, Hg., Intermedium Literatur. Beiträge zu einer Medientheorie der Literaturwissenschaft, Göttingen 2004, 27-76.

Jörg RoвERT, Einführung in die Intermedialität, Darmstadt 2014.

Jörg SCHÄFER, Sprachzeichenprozesse. Überlegungen zur Codierung von Literatur in „alten“ und „neuen“ Medien, in: Jens Schröter / Alexander Böhnke, Hg., Analog/Digital - Opposition oder Kontinuum? Zur Theorie und Geschichte einer Unterscheidung, Bielefeld 2004, 143-168.

Jens ScHRÖTER: Discourses and Models of Intermediality, in: Tötösy de Zepetnek, Digital Humanities, 32-42.

Christine SchwaneCKe, Filmic Modes in Literatur, in: Gabriele Rippl, Hg., Handbook Intermediality. Literatur Image - Sound - Music, Berlin / Boston 2015, 268-286.

Steven TötÖSY DE ZEPETNEK, Hg., Digital Humanities and the Study of Intermediality in Comparative Cultural Studies, Lavayette 2013.

Uwe WIRTH, Intermedialität, in: Alexander Roesler / Bernd Stiegler, Hg., Grundbegriffe der Medientheorie, München 2005, 114-121.

Werner WoLF, Intermedialität: Ein weites Feld und eine Herausforderung für die Literaturwissenschaft, in: Herbert Foltinek / Christoph Leitgeb, Hg., Literaturwissenschaft intermedial - interdisziplinär, Wien 2002, 165.

Werner WoLF, Towards a Functional Analysis of Intermediality. The Case of Twentieth-Century Musicalized Fiction, in: Erik Hedling / Ulla-Britta Lagerroth, Hg., Cultural Functions of Intermedial Exploration, Amsterdam / New York 2002, 15-34.

Werner Wolf, (Inter)mediality and the Study of Literature, in: Tötösy de Zepetnek, Digital Humanities, 19-31.

Evi ZemAnEK, Intermedialität - Interart Studies, in: Evi Zemanek / Alexander Nebrig, Hg., Komparatistik, Berlin 2012, 159-174. 